Research Journal of Biological Sciences 6 (1): 9-11, 2011

ISSN: $1815-8846$

(C) Medwell Journals, 2011

\title{
Phylogenetic Analysis of Lysozyme $C$ from the Scorpion Mesobuthus eupeus Venom Gland
}

\author{
${ }^{1}$ Ghafar Eskandari, ${ }^{2}$ Abbas Jolodar and ${ }^{1}$ Nabiallah Khoonmirzaei \\ ${ }^{1}$ Department of Agriculture, Islamic Azad University, Izeh Branch, Iran \\ ${ }^{2}$ Department of Basic Sciences, Faculty of Veterinary Medicine, \\ Shahid Chamran University of Ahvaz, Ahvaz, Iran
}

\begin{abstract}
Many studies have been carried out on peptides and genes encoding scorpion toxins from the venom of the scorpion Mesobuthus eupeus. The scorpion venom contains a diversity of bioactive peptides which could cause toxic effects and can be candidates for drug design and development. The anti-microbial lysozymes among them are of great value. Lysozymes are hydrolytic enzymes characterized by the ability to cleave the $\beta$ - $(1,4)$-glycosidic bond between $\mathrm{N}$-acetylmuramic acid and $\mathrm{N}$-acetyl-D-glucosamine in a peptidoglycan layer, the major bacterial cell wall polymer. The total RNA was extracted from venom glands of Mesobuthus eupeus species of Kuzestan. cDNA was synthesized with extracted total RNA as template and modified oligo (dT) as primer. In order to amplify cDNA encoding a Lys-C peptide, semi-nested RT-PCR was performed with the specific primers followed by sequencing of the amplified fragment. The full-length cDNA sequence contains a 438 nucleotide open reading frame encoding a peptide of 144 amino acids with molecular weight of $16.702 \mathrm{kDa}$. A putative 22-residue signal peptide was identified. Based on the phylogenetic tree of MesoLys-C and c-type lysozyme of East Mediterranean M. eupeus it is concluded that $M$. eupeus of Khuzestan and East Mediterranean M. eupeus belong to different subspecies.
\end{abstract}

Key words: Phylogenetic analysis, anti-microbial protein, lysozyme C, scorpion venom, amino acids, Iran

\section{INTRODUCTION}

All known scorpion species possess a venom apparatus which has been an important determinant in contributing to the successful survival of these animals for $>400$ million years. Scorpion venom is a combinatorial library of peptides and proteins which could cause toxicological responses and can be candidates for drug design and development (Menez, 1998). Several recent studies have demonstrated that scorpine-like peptides isolated from the venomous gland of some scorpion species have anti-bacterial and -malaria effects (Conde et al., 2000). These and other anti-microbial peptides found in scorpions may serve as a promising lead candidate in the development of novel antibiotic molecules. In this context, lysozymes are of great importance. Lysozymes are muramidases that damage the peptidoglycan layer of the bacterial cell wall by hydrolysing $\beta$ - $(1,4)$-glycosidic linkages between $\mathrm{N}$-acetylmuramic acid and N-acetyl-D-glucosamine residues (Gandhe et al., 2007). The known lysozymes within the animal phyla are generally classified into 3 main types; chicken-type (c-type), invertebrate-type (i-type) and goose-type (g-type) (Bachali et al., 2002). The c-type lysozyme has been found in many organisms including vira, bacteria, plants, insects, reptiles, birds and mammals (Tamura et al., 2007) including scorpions (Elgar et al., 2006). Generally, lysozymes play an important defense role in the innate immunity. However, the exact biological role of lysozymes from scorpion venoms remains to be explored as they have a relatively high expression level. In this research, researchers report the characterization and phylogenetic analysis of c-type lysozyme from the venom glands of Mesobuthus eupeus scorpions of Buthidae family which are widespread in Iran, especially in Khuzestan province.

\section{MATERIALS AND METHODS}

Scorpion samples: The specimens of $M$. eupeus were collected in Khuzestan province (Iran) and transported to the Reference Laboratory of the Razi Institute. They were killed 2 days after manual extraction of the venom to allow the toxin producing cells of the venom glands to enter into a secretory phase. Twenty separated venom glands were used for total RNA extraction.

Corresponding Author: Ghafar Eskandari, Department of Agriculture, Islamic Azad University, Izeh Branch, Iran 
Total RNA extraction: About $4 \mu \mathrm{g}$ of total RNA was extracted from the venom glands of scorpions $(0.5 \mathrm{~g}$ of tissue material) using RNA $^{\mathrm{TM}}$ (Cinagene, Iran) according to the manufacture procedure. The RNA pellets were dissolved in DEPC-ddH $\mathrm{H}_{2} \mathrm{O}$ and used for cDNA synthesis immediately.

cDNA library synthesis: The cDNA was synthesized from the extracted total RNA as template and modT (modified oligo-dT) (5'-gggtctagagctcgagtcactttttttttttt tttt-3') as primer. ModT was added to the extracted RNA and incubated at $70^{\circ} \mathrm{C}$ for $5 \mathrm{~min}$ and immediately transferred into ice for $2 \mathrm{~min}$. The mixture of $5 \times$ buffer, dNTPs, ribolock, reverse transcriptase and $\mathrm{ddH}_{2} \mathrm{O}$ was added to the samples followed by incubation at $42^{\circ} \mathrm{C}$ for $60 \mathrm{~min}$ after which the samples were incubated at $70^{\circ} \mathrm{C}$ for 10 min and immediately transferred into ice.

Semi-nested RT-PCR amplification: For the cDNA amplification, semi-nested RT-PCR technique was used. The 1st round of PCR was performed using modT-R (5'- cccagatctcgagctcagtg-3'), lys-F 5'-gcgcggat ccaagatggetttcaagtttcatt-3' primers and synthesized cDNA as template. The 2nd round of PCR was performed using lys-F and lys-R ( $5^{\prime}$-gcgcaagctttacagttgtta tcattgataaatt- $3^{\prime}$ ) primers and the PCR products of the initial amplification as templates. The PCR conditions for both rounds were as follows initial denaturation at $95^{\circ} \mathrm{C}$ ( $5 \mathrm{~min}$ ) followed by 35 cycles of denaturation at $94^{\circ} \mathrm{C}$ $(40 \mathrm{sec})$, annealing at $56^{\circ} \mathrm{C}(90 \mathrm{sec})$ and extension at $72^{\circ} \mathrm{C}$ ( $1 \mathrm{~min}$ ) with a final extension at $72^{\circ} \mathrm{C}(10 \mathrm{~min})$. Amplicons were separated by $1 \%$ agarose gel electrophoresis and visualized by UV transilluminator.

DNA sequencing and bio-informatics analysis: The amplified cDNA fragments were purified from the gel by QIAquick agarose gel extraction kit and sent to Kawsar Bio-tech company for nucleotide sequencing. Sequence similarity analysis against GenBank database entries was performed using BLAST at the NCBI website (http://www.ncbi.nlm.nih.gov). The nucleotide sequences were translated into the corresponding amino acids and the predicted signal peptide sequence was identified using online tool software at the Expasy website (http://expasy.org/tools). The sequences used for alignment and phylogenetic analysis were retrieved from SWISS-PROT database (http://www.expasy.org/sprot). The alignment was performed using the multiple sequence alignment program ClustalW 2.03 followed by manual adjustment (Thompson et al., 1997) and viewed by the Jalview software (Waterhouse et al., 2009). Phylogenetic analysis was carried out with Neighbor-joining method implemented in MEGA 3.1 (Tamura et al., 2007).

\section{RESULTS}

In order to characterize and assay the mRNAs, single strand cDNAs were synthesized and the cDNA fragments were amplified by RT-PCR technique. The length of the coding region was $438 \mathrm{bp}$, encoding a polypeptide of 144 amino acid residues with a calculated molecular weight of $16.702 \mathrm{kDa}$ and theoretical isoelectric point of 7.54. To obtain a cDNA probe for the screening of the cDNA library, researchers performed PCR which yielded a predominant $450 \mathrm{bp}$ product (Fig. 1).

From this fragment a cDNA probe sequence was determined. The precursor sequence of a 438 nucleotide open reading frame consisted of a putative 22-amino acids length signal peptide and lysine at the position $23 \mathrm{rd}$ was assumed to represent the start of the mature protein (Fig. 2). The MesoLys-C (Mesobuthus Lys-C) amino acid sequence comparison performed against GenBank NCBI database revealed that the amino acid sequence of MesoLys-C is highly homologous to c-type lysozymes from other scorpions and arthropods. To analyze the evolutionary aspects of the MesoLys-C peptide, researchers generated a phylogenetic tree encompassing known c-type lysozymes within the animal phyla; scorpion, invertebrate and vertebrate (Fig. 3).

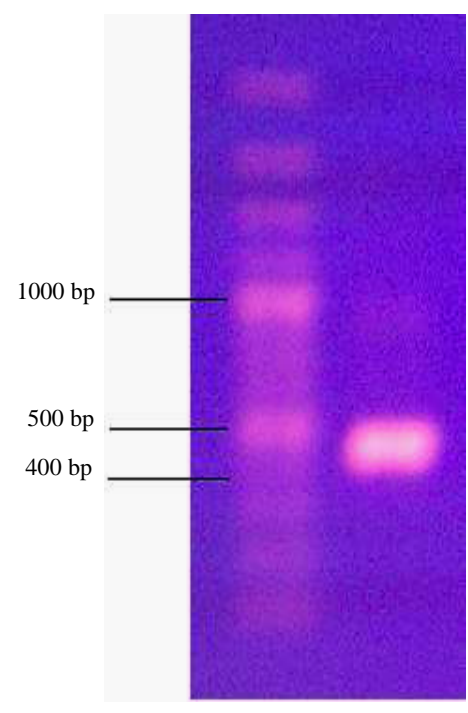

Fig. 1: PCR amplification of Lys-C cDNA from $M$. eupeus venom gland

M A F K F S F F T V L C L C V F I E N L D G R F GR CELAK LL V F N G I P Y K D P D W VCLAYYQSRLESSFMSPVSNGHR EYGIFQISSTDDNLDDDIKCAKLIH RRHKFDAWYAWKAHVKDKELSQ FINDNNCMAFKF

Fig. 2: The full-length cDNA sequence of Lys-C. The signal peptide is highlighted; the mature peptide is underlined 


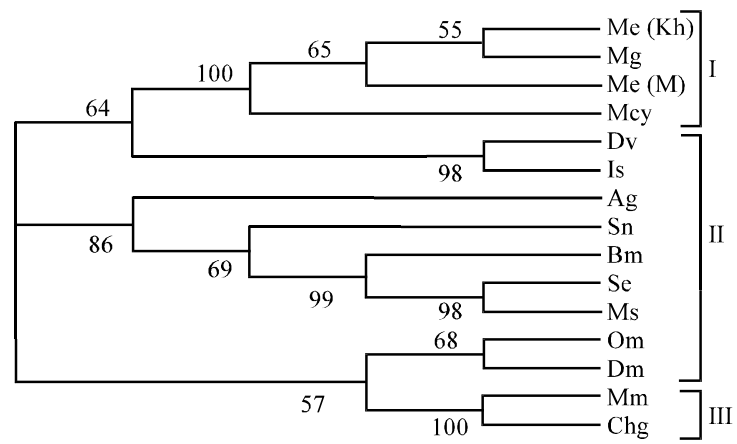

Fig. 3: Phylogenetic tree of MesoLys-C and lysozymes C from other species. $\mathrm{Me}(\mathrm{Kh})$ : Mesobuthus eupeus of Khuzestan; Mg: Mesobuthus gibbosus; $\mathrm{Me}$ (M): East Mediterranean Mesobuthus eupeus; Mcy: Mesobuthus cyprius, Dv: Dermacentor variabilis, Is: Ixodes scapularis; Ag: Anopheles gambiae; Sn: Simulium nigrimanum; Bm: Bombyx mori, Se: Spodoptera exigua; Ms: Manduca sexta; Om: Ornithodoros moubata; Dm: Drosophila melanogaster; Mm: Mus musculus; Chg: Chicken gallus. Group I: Scorpion; Group II: Invertebrate; Group III: Vertebrate. Numbers indicate bootstrap support based on 100 replicates

\section{DISCUSSION}

In this study, researchers identified and compared the MesoLys-C amino acid sequence with the representative c-type lysozymes of 3 major phyla; scorpion, invertebrate and vertebrate (Fig. 3). According to the dendrogram, all scorpion samples analyzed (M. eupeus of Khuzestan, M. gibbosus, M. cyprius and East Mediterranean M. eupeus) could be considered phylogenetically different subspecies by possessing the lysozyme $\mathrm{C}$ peptide in their venom. Moreover, MesoLysC isolated from $M$. eupeus of Khuzestan displayed the highest and the lowest sequence similarities with M. gibbosus and M. cyprius, respectively. This result is in accordance with other similar studies (Jain et al., 2001; Wang et al., 2009).

Further comparison of MesoLys-C with those from the group II and III species showed marked difference between the lysozyme $\mathrm{C}$ amino acid sequences displaying the lowest homology with the vertebtrate group. The study also revealed that the residues of catalytic site in c-type lysozyme of scorpions are different from those of chicken and other organisms.

\section{CONCLUSION}

Based on the phylogenetic tree of MesoLys-C and c-type lysozyme of East Mediterranean M. eupeus, it is said that $M$. eupeus of Khuzestan and East Mediterranean $M$. eupeus belong to different subspecies.

\section{ACKNOWLEDGEMENTS}

This research was supported by the Razi Reference Laboratory of Scorpion Research (RRLS), Iran. Researchers are grateful to Dr. Ghaemmaghami, Mr. Taghavi, Mr. Masihipour and Mr. Bahrani for their kind supports.

\section{REFERENCES}

Bachali, S., M. Jager, A. Hassanin, F. Schoentgen, P. Jolles, A. Fiala-Medioni and J.S. Deutsch, 2002. Phylogenetic analysis of invertebrate lysozymes and the evolution of lysozyme function. J. Mol. Evol., 54: $652-664$.

Conde, R., F.Z. Zamudio, M.H. Rodriguez and L.D. Possani, 2000. Scorpine, an anti-malaria and anti-bacterial agent purified from scorpion venom. FEBS Lett., 471: 165-168.

Elgar, D., J.D. Plessis and L.D. Plessis, 2006. Cysteine-free peptides in scorpion venom: Geographical distribution, structure-functionrelationship and mode of action. Afr. J. Biotechnol., 5: 2495-2502.

Gandhe, A.S., G. Janardhan and J. Nagaraju, 2007. Immune upregulation of novel antibacterial proteins from silkmoths (Lepidoptera) that resemble lysozymes but lack muramidase activity. Insect Biochem. Mol. Biol., 37: 655-666.

Jain, D., D.T. Nair, G.J. Swaminathan, E.G. Abraham, J. Nagaraju and D.M. Salunke, 2001. Structure of the induced antibacterial protein from tasar silkworm, antheraea mylitta: Implications to molecular evolution. J. Biol. Chem., 276: 41377-41382.

Menez, A., 1998. Functional architectures of animal toxins: A clue to drug design. Toxicon, 36: 1557-1572.

Tamura, K., J. Dudley, M. Nei and S. Kumar, 2007. MEGA4: Molecular evolutionary genetics analysis (MEGA) software version 4.0. Mol. Biol. Evol., 24: 1596-1599.

Thompson, J.D., T.J. Gibson, F. Plewniak, F. Jeanmougi and D.G. Higgins, 1997. The CLUSTAL X windows interface: Flexible strategies for multiple sequence alignment aided by quality analysis tools. Nucleic Acids Res., 25: 4876-4882.

Wang, W.X., Y.P. Wang, X.J. Deng, X.L. Dang and J.H. Tian et al., 2009. Molecular and functional characterization of a c-type lysozyme from the Asian corn borer, Ostrinia furnacalis. J. Insect Sci., 9: 1-13.

Waterhouse, A.M., J.B. Procter, D.M. Martin, M. Clamp and G.J. Barton, 2009. Jalview version 2-a multiple sequence alignment editor and analysis workbench. Bioinformatics, 25: 1189-1191. 